\title{
Challenges in Structural Investigation of Transient Protein-Protein Interactions
}

Kelly L Wormwood, Armand G Ngounou Wetie, Izabela Sokolowska, Alisa G Woods and Costel C Darie*

Biochemistry \& Proteomics Group, Department of Chemistry \& Biomolecular Science, Clarkson University, USA

Keywords: Mass spectrometry; Proteomics; Protein-protein interactions; Blue native PAGE

\section{Introduction}

Receptor Tyrosine Kinases (RTKs) are transmembrane proteins that, upon stimulation by their soluble ligands dimerize or multimerize and autophosphorylate themselves on the cytoplasmic side and activate signal transduction pathways, which in turn trigger either nuclear, transcriptional responses or a cellular effect such as cytoskeletal remodeling. However, while the classical RTK pathway is well

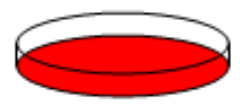

Unstimulated cells

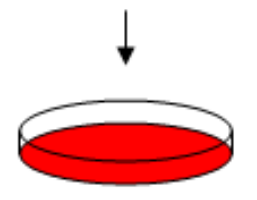

Control cells<smiles>[13CH3]</smiles>

Cell Lysis<smiles>[13CH3]</smiles>

pY99-IP<smiles>[3H][3H]</smiles>

BN-PAGE

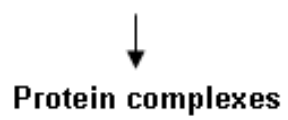

$\downarrow$

Biochemical \& Mass spectrometric characterization

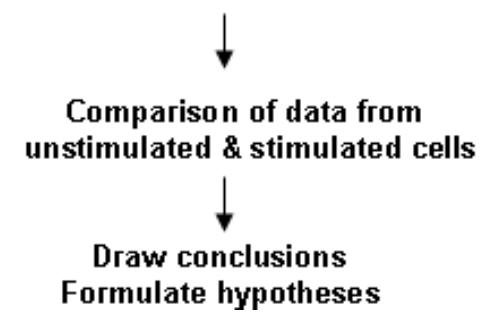

Figure 1: The workflow in a proteomics experiment for identification of stable and transient PPIs upon activation of signal transduction pathways. pY-IP: phosphotyrosine-immunopurification. Possible theoretical scenarios for analysis of protein-protein interactions, assuming a pY99-IP. understood, the intracellular protein interaction events that lead to these responses are still not yet well understood.

Upon activation of signal transduction pathways, many stable and transient Protein-Protein Interactions (PPIs) occur. However, analyzing PPIs in these signaling pathways is difficult using current methods and any improvement in this regard will help in understanding these pathways and how they function.

\section{Current Methods for Analysis of PPIs}

Analysis of PPIs in signaling pathways starts with the Immunoprecipitation (IP) of the receptors followed by identification of their protein interaction partners. In the next step, additional interacting partners may be identified [1-9]. A very common method for studying PPIs is co-Immunoprecipitation (co-IP) [10,11]. However, this method is knowledge-dependent: it may be used only if preliminary data already exists. For example, if two proteins are already known to interact with each other (i.e. interaction of two proteins is already established by a different method, such as two-hybrid screen), co-IP may be confirmative. Therefore, analysis of PPIs has been limited to co-IPs or two hybrid screens leading to the construction of interaction networks. Additional biochemical methods for identification of PPIs include electrophoresis, chromatography or mass spectrometry.

Two electrophoresis methods are of particular interest: native electrophoresis and Blue Native PAGE (BN-PAGE). In native electrophoresis, one may use regular self-made, custom gradient, PAGE, but without SDS. Having no SDS, the proteins do not denature and the PPIs are not compromised. A variation of Native electrophoresis that is used for identification of PPIs is Electrophoresis Mobility Shift Assay (EMSA), where the PPIs can be easily identified based on their shift in electrophoresis mobility. Advantage for this method is that not only PPIs, but also DNA-proteins or RNA- proteins interactions can be investigated and identified.

BN-PAGE is a relatively recently discovered method and is particularly suitable for investigation of PPIs and protein complexes [1218] (Figure 1). This method separates protein complexes based on their molecular mass, which is induced by addition of Coomassie dye to the protein sample and to the running buffer. The Coomassie dye induces a uniform external charge to the protein complexes, playing a role similar to detergent SDS (induced external charge), but without denaturing the proteins. Separation of protein complexes from the BN-PAGE gel lane

*Corresponding author: Costel C Darie, Biochemistry \& Proteomics Group Department of Chemistry \& Biomolecular Science, Clarkson University, 8 Clarkson Avenue, Potsdam, NY, 13699-5810, USA, Tel: 315-268-7763; Fax: 315-268-6610; E-mail: cdarie@clarkson.edu

Received November 29, 2013; Accepted January 22, 2014; Published January 24, 2014

Citation: Wormwood KL, Wetie AGN, Sokolowska I, Woods AG, Darie CC (2014) Challenges in Structural Investigation of Transient Protein-Protein Interactions. Mod Chem appl 2: 117. doi:10.4172/2329-6798.1000117

Copyright: @ 2014 Wormwood KL, et al. This is an open-access article distributed under the terms of the Creative Commons Attribution License, which permits unrestricted use, distribution, and reproduction in any medium, provided the original author and source are credited. 
$A$

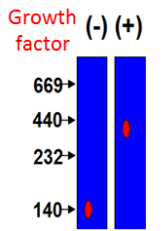

\section{B} $(-)(+)$

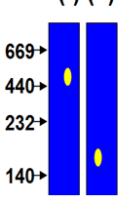

C $(-)(+)$

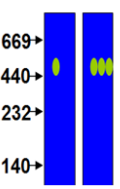

\section{D} $(-)(+)$

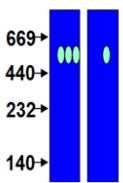

Figure 2: Possible theoretical scenarios for analysis of protein-protein interactions, assuming a pY99-IP. A: One protein interacts with other proteins upon stimulation. B: One protein complex is disassembled upon stimulation. C: One protein complex becomes over-phosphorylated upon stimulation. D: One protein complex is over-dephosphorylated upon stimulation. E: A protein complex is completely dephosphorylated (green) or phosphorylated (yellow) upon stimulation.

separated in the first dimension (1D) in a second dimension (2D) SDSPAGE followed by Coomassie staining or by Western Blotting (WB) may reveal the subunit composition of a particular protein complex, as well as the interacting partners of a particular protein. Additional protein complexes may also be identified (Figure 2). A variant of BN-PAGE is Colorless Native PAGE or Clear Native PAGE (CN-PAGE), in which the proteins are separated just like in BN-PAGE, except that the Coomassie dye is not included. As a consequence, the protein complexes are separated according to their internal charge and independent of their molecular mass. A particularly useful combination includes CN-PAGE with SDS-PAGE in 2D-PAGE followed by SDS-PAGE and Coomassie staining or SDS-PAGE and Western blotting, similar to BN-PAGE described earlier. In addition, combination of CN-PAGE and BN-PAGE is very useful and unique for the separation of protein complexes with identical molecular mass, but different internal charge. For example, when two protein complexes with identical mass are separated by BN-PAGE, their subunit composition cannot be identified, unless the subunit composition of the protein complexes investigated is known. However, if not known, the protein complexes can be first separated by CN-PAGE (according to their internal charge) and then by $\mathrm{BN}$ PAGE (according to their molecular mass). Therefore, the end result is complete separation of two protein complexes with identical mass, but different internal charge.

Mass spectrometry (MS) is one of the most powerful tools for identifying proteins [2,19-34] and PPIs [1,3,4,10,14,35,36], simply because it can be easily combined with IP, SDS-PAGE, chromatography, native PAGE, BN-PAGE CN-PAGE, and allows one to identify many potential interacting partners of a protein by analyzing the proteins or protein complexes (isolated and/or purified by one of the previously described methods) by Matrix Assisted Laser Desorption Ionization Mass Spectrometry (MALDI-MS) or by liquid chromatographytandem mass spectrometry (LC-MS/MS), in an approach referred to as proteomics [3-5,14,29,30,36-42]. As such, most PPIs are indirectly identified through digestion of the protein complexes purified through one of the previously described methods (i.e. IP) followed by MS analysis, which leads to identification of the peptides that are part of proteins that can interact with each other. This approach is also called bottom up proteomics and may also be called bottom-up PPIs proteomics. The opposite method for bottom-up PPIs proteomics is top-down PPIs proteomics, where intact protein complexes are analyzed by MS without prior digestion.

\section{Technical Limitations}

Current methodology used for identification of the PPIs could definitely use some improvements. For example, in experiments in which a particular protein is identified as a participant in signal transduction pathways, no functional assignment is made to it unless its post-translational modifications (such as phosphorylation) are dramatically increased or decreased. Furthermore, even when the interacting partners of the proteins involved in signaling are identified and characterized, the functional significance of newly formed PPIs via ligand stimulation is still missed. Therefore, any optimization of the current technology will be a great improvement. Analysis of protein complexes from signal transduction pathways in terms of size, composition, post-translation modifications, multimerization level, and abundance would also benefit from the introduction of new methods.

Affinity or immunoaffinity purification of protein complexes followed by SDS-PAGE and MALDI-MS or LC-MS/MS is definitely the most frequently used method for identification of PPIs. However, the biggest problem with this approach is that it cannot be adapted for large scale identification of PPIs. Therefore, this approach is not eligible for high throughput identification of PPIs (hopefully in the near future).

BN-PAGE is a good alternative option for simultaneous identification of protein complexes and PPIs. However, although it is a useful method, BN-PAGE has many downsides. A potential problem for BN-PAGE is that protein complexes that have identical molecular mass may co-migrate and subunits from two different protein complexes may be falsely identified as being part of the same protein complex. This pitfall may be solved by adding for those particular protein complexes a pre-purification step to BN-PAGE, known as CN-PAGE. Separation of the gel lane that results from the second dimension in a third dimension (SDS-PAGE) will reveal the subunit composition of each protein complex. Another potential problem includes difficulties in the identification of a protein that is part of a protein complex in BN-PAGE (1D) using Western blotting experiments. This problem may occur because many commercial antibodies are made against only one epitope of a protein and if the epitope is buried inside the protein or protein complex, it may not be identified, thus leading to false negative data. This may happen mainly because the proteins and protein complexes are separated under native conditions that preserve their normal conformation.

MALDI-MS and LC-MS/MS are a good option for identification of the PPIs and scientists from many labs succeeded in analyzing both stable and transient PPIs by their direct analysis. Both MALDI-MS and LC-MS/MS (or electrospray ionization mass spectrometry or ESI-MS, a method that does not use HPLC combined with a mass spectrometer) have been used successfully to analyze and identify individual PPIs. However, we already have the instrumentation required for analysis of PPIs (both MALDI-MS and ESI-MS-based instrumentation), these methods have not yet been used in large scale, high-throughput analysis of PPIs for two reasons: 1) the protein subunits of these PPIs have functional post-translational modifications (PTMs) such as phosphorylation and one modification gives the PPIs a function, while removal of the phosphoryl group will give the PPIs a different function. 2) the second and the most important reason is the still weak bioinformatic support in analysis of PPIs. Even if we have the instruments and expertise to investigate PPIs, we still do not have yet the bioinformatics power to successfully identify the PPIs on a large scale and in high throughput.

\section{Perspectives}

Developing new functional proteomics methodology which, in addition to identifying and quantifying proteins, also gives us information about the protein complexes that contain a particular protein and about the size and subunit composition of that particular 
protein complex will be a great advancement. Successful establishment of such a method for functional proteomics will hopefully also provide a powerful new approach for studying other key signal transduction pathways. The method could be used to build a comprehensive PPI network involved not only in RTKs pathways, but also in other pathways and stable and transient PPIs. This method is yet to come.

\section{Acknowledgement}

This work was in part supported by the David A. Walsh '67 Fellowship, awarded to KLW \& CCD. This work was also supported in part by Mary Joyce, Robert Matloff, Ken Sandler, Bob and Karen Brown, Bonhomie Wine Imports, Wolverine Packing Co. and by the SciFund challenge contributors.

\section{References}

1. Darie CC (2013) Investigation of Protein-Protein Interactions by Blue NativePAGE \& Mass Spectrometry. Mod Chem appl 1: e111.

2. Florian PE, Macovei A, Lazar C, Milac AL, Sokolowska I, et al. (2013) Characterization of the anti-HBV activity of HLP1-23, a human lactoferrinderived peptide. J Med Virol 85: 780-788.

3. Ngounou Wetie AG, Sokolowska I, Woods AG, Roy U, Deinhardt K, et al. (2014) Protein-protein interactions: switch from classical methods to proteomics and bioinformatics-based approaches. Cell Mol Life Sci 71: 205-228.

4. Ngounou Wetie AG, Sokolowska I, Woods AG, Roy U, Loo JA, et al. (2013) Investigation of stable and transient protein-protein interactions: Past, present and future. Proteomics 13: 538-557

5. Petrareanu C, Macovei A, Sokolowska I, Woods AG, Lazar C, et al. (2013) Comparative proteomics reveals novel components at the plasma membrane of differentiated HepaRG cells and different distribution in hepatocyte- and biliary-like cells. PLoS One 8: e71859.

6. Sokolowska I, Ngounou Wetie AG, Woods AG, Darie CC (2012) Automatic determination of disulfide bridges in proteins. J Lab Autom 17: 408-416.

7. Guerrera IC, Kleiner O (2005) Applications of mass spectrometry in proteomics Biosci Rep 25: 71-93.

8. Sokolowska I, Woods AG, Gawinowicz MA, Roy U, Darie CC (2012) Identification of potential tumor differentiation factor (TDF) receptor from steroid-responsive and steroid-resistant breast cancer cells. J Biol Chem 287 1719-1733.

9. Sokolowska I, Woods AG, Gawinowicz MA, Roy U, Darie CC (2013) Characterization of tumor differentiation factor (TDF) and its receptor (TDF-R). Cell Mol Life Sci 70: 2835-2848.

10. Roy U, Sokolowska I, Woods AG, Darie CC (2012) Structural investigation of tumor differentiation factor. Biotechnol Appl Biochem 59: 445-450.

11. Woods AG, Sokolowska I, Darie CC (2012) Identification of consistent alkylation of cysteine-less peptides in a proteomics experiment. Biochem Biophys Res Commun 419: 305-308.

12. Darie CC, Biniossek ML, Winter V, Mutschler B, Haehnel W (2005) Isolation and structural characterization of the Ndh complex from mesophyll and bundle sheath chloroplasts of Zea mays. FEBS J 272: 2705-2716.

13. Darie CC, Janssen WG, Litscher ES, Wassarman PM (2008) Purified trout egg vitelline envelope proteins VEbeta and VEgamma polymerize into homomeric fibrils from dimers in vitro. Biochim Biophys Acta 1784: 385-392.

14. Darie CC, Shetty V, Spellman DS, Zhang G, Xu C, et al. (2008) Blue Native PAGE and mass spectrometry analysis of the ephrin stimulation- dependen protein-protein interactions in NG108-EphB2 cells. Applications of Mass Spectrometry in Life Safety, NATO Science for Peace and Security Series A: Chemistry and Biology 3-22.

15. Litscher ES, Janssen WG, Darie CC, Wassarman PM (2008) Purified mouse egg zona pellucida glycoproteins polymerize into homomeric fibrils under nondenaturing conditions. J Cell Physiol 214: 153-157.

16. Schagger $H$, Cramer WA, von Jagow G (1994) Analysis of molecular masses and oligomeric states of protein complexes by blue native electrophoresis and isolation of membrane protein complexes by two-dimensional native electrophoresis. Anal Biochem 217: 220-230.

17. Schagger $H$, von Jagow $G$ (1991) Blue native electrophoresis for isolation of membrane protein complexes in enzymatically active form. Anal Biochem 199: 223-231.
18. Woods AG, Sokolowska I, Yakubu R, Butkiewicz M, LaFleur M, et al. (2011) Blue native page and mass spectrometry as an approach for the investigation of stable and transient protein-protein interactions. Oxidative Stress: Diagnostics, Prevention, and Therapy 1083: 341-367.

19. Aebersold R, Mann M (2003) Mass spectrometry-based proteomics. Nature 422: 198-207.

20. Blagoev B, Kratchmarova I, Ong SE, Nielsen M, Foster LJ, et al. (2003) A proteomics strategy to elucidate functional protein-protein interactions applied to EGF signaling. Nat Biotechnol 21: 315-318.

21. Darie CC, De Pascalis L, Mutschler B, Haehnel W (2006) Studies of the Ndh complex and photosystem II from mesophyll and bundle sheath chloroplasts of the C4-type plant Zea mays. J Plant Physiol 163: 800-808.

22. Gygi SP, Rist B, Gerber SA, Turecek F, Gelb MH, et al. (1999) Quantitative analysis of complex protein mixtures using isotope-coded affinity tags. Nat Biotechnol 17: 994-999.

23. Ngounou Wetie AG, Sokolowska I, Woods AG, Wormwood KL, Dao S, et al. (2013) Automated mass spectrometry-based functional assay for the routine analysis of the secretome. J Lab Autom 18: 19-29.

24. Ong SE, Blagoev B, Kratchmarova I, Kristensen DB, Steen H, et al. (2002) Stable isotope labeling by amino acids in cell culture, SILAC, as a simple and accurate approach to expression proteomics. Mol Cell Proteomics 1: 376-386.

25. Han X, Aslanian A, Yates JR $3^{\text {rd }}$ (2008) Mass spectrometry for proteomics. Curr Opin Chem Biol 12: 483-490.

26. Petrareanu C, Macovei A, Sokolowska I, Woods AG, Lazar C, et al. (2013) Comparative proteomics reveals novel components at the plasma membrane of differentiated HepaRG cells and different distribution in hepatocyte- and biliary-like cells. PLoS One 8: e71859.

27. Shevchenko A, Wilm M, Vorm O, Mann M (1996) Mass spectrometric sequencing of proteins silver-stained polyacrylamide gels. Anal Chem 68: 850858.

28. Spellman DS, Deinhardt K, Darie CC, Chao MV, Neubert TA (2008) Stable isotopic labeling by amino acids in cultured primary neurons: application to brain-derived neurotrophic factor-dependent phosphotyrosine-associated signaling. Mol Cell Proteomics 7: 1067-1076.

29. Steen H, Kuster B, Fernandez M, Pandey A, Mann M (2002) Tyrosine phosphorylation mapping of the epidermal growth factor receptor signaling pathway. J Biol Chem 277: 1031-1039.

30. Wang Y, Li R, Du D, Zhang C, Yuan H, et al. (2006) Proteomic analysis reveals novel molecules involved in insulin signaling pathway. J Proteome Res 5: 846855

31. Zhang G, Fenyö D, Neubert TA (2008) Screening for EphB signaling effectors using SILAC with a linear ion trap-orbitrap mass spectrometer. J Proteome Res 7: 4715-4726.

32. Zhang G, Neubert TA (2006) Automated comparative proteomics based on multiplex tandem mass spectrometry and stable isotope labeling. Mol Cell Proteomics 5: 401-411.

33. Zhang G, Spellman DS, Skolnik EY, Neubert TA (2006) Quantitative phosphotyrosine proteomics of EphB2 signaling by stable isotope labeling with amino acids in cell culture (SILAC). J Proteome Res 5: 581-588.

34. Zhang G, Ueberheide BM, Waldemarson S, Myung S, Molloy K, et al. (2010) Protein quantitation using mass spectrometry. Methods Mol Biol 673: 211-222.

35. Alastair Aitken (2005) Identification of post-translational modifications by mass spectrometry. The Proteomics Protocols Handbook 431-437.

36. Sokolowska I, Gawinowicz MA, Ngounou Wetie AG, Darie CC (2012) Disulfide proteomics for identification of extracellular or secreted proteins. Electrophoresis 33: 2527-2536.

37. Sokolowska I, Dorobantu C, Woods AG, Macovei A, Branza-Nichita N, et al. (2012) Proteomic analysis of plasma membranes isolated from undifferentiated and differentiated HepaRG cells. Proteome Sci 10: 47.

38. Sokolowska I, Ngounou Wetie AG, Roy U, Woods AG, Darie CC (2013) Mass spectrometry investigation of glycosylation on the NXS/T sites in recombinant glycoproteins. Biochim Biophys Acta 1834: 1474-1483.

39. Sokolowska I, Woods AG, Gawinowicz MA, Roy U, Darie CC (2012) Identification of a potential tumor differentiation factor receptor candidate in prostate cancer cells. FEBS J 279: 2579-2594. 
Citation: Wormwood KL, Wetie AGN, Sokolowska I, Woods AG, Darie CC (2014) Challenges in Structural Investigation of Transient Protein-Protein Interactions. Mod Chem appl 2: 117. doi:10.4172/2329-6798.1000117

40. Sokolowska I, Woods AG, Wagner J, Dorler J, Wormwood K, et al. (2011) Mass spectrometry for proteomics-based investigation of oxidative stress and heat shock proteins. Oxidative Stress: Diagnostics, Prevention, and Therapy 1083: 369-411.

41. Thome J, Coogan AN, Woods AG, Darie CC, Häßler F (2011) CLOCK Genes and Circadian Rhythmicity in Alzheimer Disease. J Aging Res 2011: 383091.
42. Darie CC, Litscher ES, Wassarman PM (2008) Structure, processing, and polymerization of rainbow trout egg vitelline envelope proteins. Applications of Mass Spectrometry in Life Safety 23-36. 